\title{
The Osmotic Coefficient of Rod-like Polyelectrolytes: \\ Computer Simulation, Analytical Theory, and Experiment
}

\author{
M. Deserno ${ }^{1}$, C. Holm ${ }^{1}$, J. Blaul ${ }^{2}$, M. Ballauff ${ }^{2}$, and M. Rehahn ${ }^{3}$ \\ ${ }^{1}$ Max-Planck-Institut für Polymerforschung, Ackermannweg 10, 55128 Mainz, \\ Germany \\ ${ }^{2}$ Polymer-Institut, Universität Karlsruhe, Kaiserstraße 12, 76128 Karlsruhe, \\ Germany \\ ${ }^{3}$ Institut für Makromolekulare Chemie, Technische Universität Darmstadt, \\ Petersenstraße 22, 64287 Darmstadt, Germany
}

\begin{abstract}
The osmotic coefficient of solutions of rod-like polyelectrolytes is considered by comparing current theoretical treatments and simulations to recent experimental data. The discussion is restricted to the case of monovalent counterions and dilute, salt-free solutions. The classical PoissonBoltzmann solution of the cell model correctly predicts a strong decrease in the osmotic coefficient, but upon closer look systematically overestimates its value. The contribution of ion-ion-correlations are quantitatively studied by MD simulations and the recently proposed DHHC theory. However, our comparison with experimental data obtained on synthetic, stiff-chain polyelectrolytes shows that correlation effects can only partly explain the discrepancy. A quantitative understanding thus requires theoretical efforts beyond the restricted primitive model of electrolytes.
\end{abstract}

\section{Introduction}

Polyelectrolytes are macromolecules carrying charged groups which in polar solvents can dissociate into a highly charged macroion and oppositely charged small counterions. ${ }^{1-5}$ The high electric field of the macroion strongly couples to the counterions, which in turn will tend to partly neutralize the macroion. This phenomenon has been termed "counterion condensation". ${ }^{6-8}$ Since polyelectrolytes present an ubiquitous class of materials, the quantitative understanding of counterion condensation is of fundamental importance.

The strong correlation of the counterions with the macroion reduces their thermodynamic activity. Only a certain fraction of ions will be osmotically active and contribute to the osmotic pressure $\Pi$ of polyelectrolytes in dilute solutions, as Kern was able to show by osmotic measurements as early as $1938 .^{9}$ 
The reduced activity can be expressed in terms of the osmotic coefficient

$$
\phi:=\frac{\Pi}{\Pi_{\mathrm{id}}},
$$

where $\Pi_{i d}$ is the ideal osmotic pressure calculated for a solution of counterions interacting neither with themselves nor with the macroion. A great number of experimental studies of $\phi$ for strongly charged polyelectrolytes is now available in literature, demonstrating that $\phi$ is of the order of 0.2 to 0.3 for univalent counterions in the dilute regime. ${ }^{1-3,10-17}$ Furthermore, a comparison of experimental data obtained for different counterions show that specific interactions between counterions and the macroion can lead to an additional reduction of the measured osmotic pressure. ${ }^{1,3}$ In case of monovalent counterions, these specific effects are small as compared to the effect of counterion condensation that presents the dominant feature of strongly charged polyelectrolytes. A detailed discussion of this problem has been given by Mandel. ${ }^{1}$ However, no quantitative conclusions could be drawn from this comparison so far, since practically all experimental data have been obtained from solutions of flexible polyelectrolytes, which change their conformation upon lowering the ionic strength. ${ }^{18}$ The intricate connection of the osmotic coefficient with the conformation of the macroion is not yet fully understood, therefore studies of flexible polyelectrolytes in solution cannot lead to direct conclusions on the validity of theories developed for rod-like macroions.

In this paper we wish to discuss recent theoretical models of rod-like polyelectrolytes and compare the calculated osmotic coefficient to results of experimental investigations of a suitable model system. We restrict the discussion to the case of rod-like macroions for which a partial counterion condensation occurs, ${ }^{6,19-21}$ i.e., part of the counterions remain within a finite distance to the macroion and will not be diluted away when lowering the concentration to extremely low values. The paper is organized as follows: First the simplified situation of the cylindrical cell model and its solution within the framework of Poisson-Boltzmann (PB) theory will be discussed. Next we consider theoretical refinements designed to overcome limitations inherent to the cell model or its mean-field PB solution. In particular, computer simulations will be used to assess the importance of correlations neglected in the PB approach. Finally recent measurements of the osmotic coefficient for a synthetic rod-like polyelectrolyte will be quantitatively compared to two theoretical models and computer simulations. Hence, the present comparison allows to assess to what extend the deviations between measurements and PB theory can be accounted for by the modern theoretical approaches. 


\section{The cell model}

The cell model is a commonly used way of reducing the complicated many-body problem of a polyelectrolyte solution to an effective one-particle theory, i.e., the case of a single polyelectrolyte chain in a cell. ${ }^{3-5,19,20,22}$ The idea is to partition the solution into sub-volumes, each containing only a single macroion together with its counterions. Since each sub-volume is electrically neutral, the electric field will on average vanish on the cell surface. By virtue of this construction different sub-volumes are electrostatically decoupled to a first approximation. One may thus hope to factorize the partition function and reduce the problem to the treatment of just one sub-volume, called "cell". Its shape is assumed to reflect the symmetry of the polyelectrolyte. For a solution of rod-like polyelectrolytes with density $\rho_{\mathrm{p}}$ and rod length $L$ this gives a cylindrical cell with the radius $R$ being fixed by the condition $\pi R^{2} L \times \rho_{\mathrm{p}}=1$. The theoretical treatment is much simpler after neglecting end effects at the cylinder caps. This is equivalent to taking $L=\infty$ after mapping to the correct density.

The ions will be described as point-like, but they will electrostatically interact with the macroion as well as with each other. Their positions may thus be strongly correlated. The solvent molecules are not explicitly taken into account; rather, they are assumed to form a continuous dielectric background which is completely specified by its dielectric constant $\varepsilon_{\mathrm{r}}$.

In this simplified situation it can be proved rigorously that the osmotic pressure is given by the counterion density at the cell boundary times the thermal energy $k_{\mathrm{B}} T .^{23}$ The osmotic coefficient is thus given by the ratio of the ion density at the cell boundary divided by the average ion density.

\section{Poisson-Boltzmann theory for the cylindrical cell model}

The analytical description taking into account all individual counterions turns out to be still too involved, since - as usual - inter-ionic correlations complicate matters. The standard way out is neglecting those correlations in a mean-field spirit, as is done in the Poisson-Boltzmann description: The ionic degrees of freedom are replaced by a cylindrical counterion density, and their interaction is approximated by the assumption that the density is locally proportional to the Boltzmann-factor, see e.g. Refs.. ${ }^{19-21,24}$ It can be shown that on this meanfield level the osmotic coefficient is still given by the ratio between boundary density and average density. ${ }^{25}$

Let us briefly recapitulate the Poisson-Boltzmann treatment of the cell model. Consider a rod of radius $r_{0}$ and line charge density $\lambda>0$ coaxially enclosed in a cylinder of radius $R$. Electroneutrality is achieved by adding the appropriate amount of monovalent counterions; no additional salt shall be present. The 
strength of the electrostatic interactions is conveniently expressed by the Bjerrum length

$$
\ell_{\mathrm{B}}=\frac{e_{0}^{2}}{4 \pi \varepsilon_{0} \varepsilon_{\mathrm{r}} k_{\mathrm{B}} T},
$$

where $e_{0}$ is the unit charge, $\varepsilon_{\mathrm{r}}$ is the dielectric constant of the solvent, and $\varepsilon_{0}$, $k_{\mathrm{B}}$ and $T$ have their usual meaning. This definition suggests a dimensionless way of measuring the line charge density of the rod via the charge parameter $\xi$ :

$$
\xi=\lambda \ell_{\mathrm{B}} / e_{0} .
$$

It counts the number of unit charges on the rod per Bjerrum length and is usually called the Manning parameter. In the following only the strongly charged case $\xi>1$ will be discussed.

Within the framework of PB theory the osmotic coefficient turns out to be given by the expression ${ }^{25}$

$$
\phi_{\mathrm{PB}}=\frac{1+\gamma^{2}}{2 \xi},
$$

where the dimensionless constant of integration $\gamma$ is the solution of the transcendental equation

$$
\gamma \ln \frac{R}{r_{0}}=\arctan \frac{1}{\gamma}+\arctan \frac{\xi-1}{\gamma} .
$$

In the limit of infinite dilution the cell radius $R$ tends to infinity, which implies $\gamma \rightarrow 0$. Note that for $\gamma \rightarrow 0$ and $\xi>1$ the right hand side of Eqn. (5) tends to the constant $\pi$, hence the osmotic coefficient as computed by PB theory (logarithmically) converges to the well known Manning limiting law $\phi_{\infty}=1 / 2 \xi$. At finite densities it is always larger, however.

\section{Beyond the Poisson-Boltzmann solution of the cell model}

The theoretical treatment discussed above suffers from various approximations. First, the cell model itself is a simplified representation of the polyelectrolyte solution: It neglects rod-rod-interactions, it is incapable of describing effects due to the finite length of the rods, and it reduces the solvent to a dielectric continuum. Second, the mean-field approach discards any inter-ionic correlations which can modify the average charge distribution. In this section we briefly outline a few theoretical approaches which try to provide an improved description of the physical situation. Our main purpose is to indicate, in which direction changes of the osmotic coefficient are to be expected. 


\subsection{Integral equations}

The key approximation in Poisson-Boltzmann theory is the neglect of ionic correlations by assuming that all $N$-particle distribution functions entirely factorize. Hence, each ion "sees" only a mean ion density but no individual particles in its vicinity. However, the local ionic order around each ion influences its energy. Recall for instance that the mean charge density around an ion in a crystal is zero, but nevertheless the electrostatic energy per ion is (i) negative, and (ii) the Madelung constant depends on the crystal structure.

Integral equation theories try to explicitly compute higher order correlation functions and their impact on the one particle distribution function, i.e., the density. The problem of the electric double layer has been treated frequently in the past for various geometries. ${ }^{26-28}$ What concerns us here is that the screening of the macroion by the small counterions is found to be enhanced. Since a larger number of ions close to the rod implies a smaller number of ions at the cell boundary, this effect will lower the osmotic coefficient as compared to Poisson-Boltzmann theory.

\subsection{Correlation corrected density functionals}

The correlations neglected in the mean field approach can in principle be recaptured by adding a correction to the free energy functional corresponding to Poisson-Boltzmann theory. ${ }^{29-31}$ A local and stable functional based essentially on a Debye-Hückel solution of the one component plasma has recently been suggested by Barbosa et al. ${ }^{32}$ It leads to an enhanced counterion condensation in the vicinity of the macroion in very good quantitative agreement with Molecular Dynamics simulations. This again implies a lower density at the outer cell radius and thus a smaller osmotic coefficient. The extended free energy functional translates to a differential equation more complicated than the PB equation. However, the equilibrium ion profiles can be conveniently determined by directly implementing the functional minimization problem along the lines suggested in Ref.. ${ }^{33}$ A comparison of Poisson-Boltzmann theory, computer simulations and experiments with this correlation corrected theory will be presented below.

\subsection{Debye-Hückel-Bjerrum theory}

An attempt to go beyond the cell model with the aim of incorporating rod-rodinteractions has been suggested by Kuhn et al. ${ }^{34}$ A solution of charged rods is investigated within the Debye-Hückel-Bjerrum theory, which uses the linearized form of the Poisson-Boltzmann equation to solve for the charge distributions. The neglected nonlinearities are approximately recaptured by the explicit introduction of clusters consisting of a rod and condensed counterions. Minimizing the free energy of the system, which is constructed as a sum of electrostatic 
and entropic terms, gives the cluster distribution and thermodynamic quantities like the osmotic pressure. No cell-description is used, rather, the interaction between individual rods is explicitly computed and their mutual orientations is averaged over. For the computation of the screened rod-potential (and at a few other stages) end effects are neglected, which in the dilute limit entails the Manning limiting behavior of infinite rods. For the case of added salt the theory predicts an osmotic coefficient even below the Manning predictions, but it is unfortunately not straightforward to extend it to the salt-free case. A simpler version for the salt free case ${ }^{35}$ that neglects rod-rod interactions, again shows the osmotic coefficient to be always below the Manning limiting value. This, and the fact that it decreases upon increasing polyelectrolyte concentration, raises concerns as to whether the employed approximations lead to a well-controlled improvement beyond the cell model.

\subsection{Finite rods}

An alternative theory beyond the cell model has been put forward by Nyquist et al. ${ }^{36}$ Here the aim is to explicitly account for the finite length of the rods. It is argued that in the limit of infinite dilution a finite rod will not produce Manning condensation and the ions will become free. This implies the correct limiting osmotic coefficient of 1 instead of $1 / 2 \xi$ at zero density. At finite density the condensation is approximated by a two state model (ions are either free or condensed, and each rod carries the same fraction of condensed ions). The partition function is computed in a random phase approximation, which finally gives the equilibrium condensed fraction as a function of density. In the dilute regime this fraction is found to increase with density and the osmotic coefficient is correspondingly found to decrease. Furthermore, the calculated osmotic coefficient is larger than the Poisson-Boltzmann result. This is in strong contrast to any solution of the problem which assumes infinite rods.

Up to now there is no systematic comparison of theory and experiment, however, the model seems to overestimate considerably the osmotic coefficient when compared to experimental data. ${ }^{10,17}$ Possible reasons for this shortcoming may be related to the two state model or the random phase approximation employed in Ref.. ${ }^{36}$

\section{Simulations}

After restricting to the simplified models mentioned above, simulations can be very successfully used to test the available theories, demarcate their range of applicability, and indicate the trends of deviations which are to be expected. Consequently, versions of the cylindrical cell model have been used several times in the past as a basis for simulations. ${ }^{24,37-39}$ An important observation has been that condensation is generally enhanced compared to Poisson-Boltzmann theory, 
which is frequently attributed to the correlations neglected by the latter.

In this article we use molecular dynamics simulations to determine the osmotic coefficient for a cell model, the parameters of which have been mapped to a specific polyelectrolyte: poly(para-phenylene) (PPP, see Fig. 1 and below). We take a cubic simulation box of length $L_{\mathrm{b}}$, one charged rod parallel to an edge, and the correct number of monovalent counterions which leave the system electrostatically neutral. The ions interact via a purely repulsive Lennard-Jones potential, ${ }^{18}$ giving them a diameter of $4.4 \AA$. For the rod-ion-interaction a similar potential was used, in which the hard core was shifted to larger radii, such that the distance of closest approach between an ion and the rod was $7 \AA$. This value has been inferred from an analysis of small angle (neutron or X-ray) scattering experiments on PPP. ${ }^{40-42}$ Each ion carries a negative unit charge in its center and the rod carries a sequence of positive unit charges along its axis at a distance of $2.15 \AA$. Together with a Bjerrum length of $7.31 \AA$ (corresponding to water at $40{ }^{\circ} \mathrm{C}$ ) this gives a charge parameter $\xi=3.4$. After switching on periodic boundary conditions this geometry yields an infinite array of infinitely long rods sitting on a square lattice. When comparing to the cylindrical cell model we will map the rectangular cell belonging to one such rod with a cylindrical cell of radius $R=L_{\mathrm{b}} / \sqrt{\pi}{ }^{43}$

The electrostatic interactions in this periodic boundary geometry were computed with the help of $\mathrm{P}^{3} \mathrm{M}$ routines, ${ }^{44}$ and a Langevin thermostat ${ }^{45}$ combined with a velocity-Verlet-integrator ${ }^{46}$ (with time-step 0.01 in Lennard-Jones units) was implemented to drive the system into the canonical state. The saturation of the electrostatic energy was used to test for equilibration. A more detailed description can be found in. ${ }^{47}$ We simulated the rods at a monovalent counterion concentration of $2.18,6.79$, and $10.2 \mathrm{mmol} / \mathrm{l}$, with a number of $4.5,1.8$, and 1.2 million MD steps, respectively. The corresponding number of monovalent ions in the simulation cell were 277,1256 , and 1024 . The osmotic pressure was taken as the average of the $x x$ - and $y y$-component of the stress tensor, given that the rod points along the $z$-axis.

\section{Experiment}

From its derivation it is obvious that the cylindrical cell-model can be valid only if the macroions are sufficiently stretched. DNA in salt-free solution can be regarded as such a model polyelectrolyte. ${ }^{10,41,48}$ However, the stability of the helical conformation is severely impeded if the ionic strength is low, and measurements in salt-free solution must be carried out at low temperature to avoid the melting of the helix. To the author's best knowledge the study of Auer and Alexandrowicz ${ }^{10}$ has been the only one in which strictly salt-free solutions of DNA have been studied. A more recent investigation employed solutions of DNA with additional $2 \mathrm{mM}$ and $10 \mathrm{mM}$ added salt. ${ }^{48}$ However, in this case

counterions and salt ions will establish a Donnan equilibrium, ${ }^{2,3,49}$ which results 
in a drastic reduction of the small-ion contribution to the pressure. Its dominant origin at low polyelectrolyte density is not yet fully understood. ${ }^{50}$

Recently, an investigation of the osmotic coefficient of a synthetic rod-like polyelectrolyte has been presented. ${ }^{17}$ The system studied there consists of a poly(p-phenylene) (PPP) backbone to which positively charged side-chains have been attached (see Fig. 1). The fully aromatic backbone exhibits a high stiffness with a persistence length of approximately $22 \mathrm{~nm} .{ }^{51}$ The number-average contour length of PPP-macroions used in the experiment is of the same order. Hence, the PPP-polyelectrolytes may be treated as rod-like in very good approximation. The system has a charge parameter $\xi=3.4$ of similar magnitude as DNA $(\xi=4.2)$. The excellent chemical stability of the PPP-chains allows to study these polyelectrolytes in salt free solutions without any problem.

In Ref. ${ }^{17}$ the osmotic coefficient of the PPP system was measured for two kind of counterions, iodine and chlorine. Both sets of experimental data, which have been obtained in virtually salt free solution, clearly indicate that the Poisson-Boltzmann solution of the cell-model makes a fairly good prediction for the osmotic coefficient, but systematically overestimates it upon closer look. This is also in agreement with the earlier results obtained on DNA by Auer and Alexandrowicz. ${ }^{10}$ Moreover, it became apparent in this study that there are specific interactions of the counterions with the macroion, which can be as large as the differences from the Poisson-Boltzmann solution itself. This was concluded from the fact that chlorine counterions lead to a considerably smaller $\phi$ than iodine counterions. Such specific interactions have been found for many flexible polyelectrolytes and extensively discussed in the earlier literature. ${ }^{1,3}$ The errors in the experiment are hard to estimate, but are on the order of $7 \%$.

\section{Comparison of theory, simulations and exper- iment}

It has to be understood that an analysis of the osmotic coefficient $\phi$ measured in salt free solutions within the framework of the Poisson-Boltzmann solution of the cylindrical cell model proceeds virtually without adjustable parameters. Here, $\phi$ is solely determined by the charge parameter $\xi$, which is fixed by chemistry, the rod radius $r_{0}$, which has been measured experimentally, and the polyelectrolyte concentration, which is fixed by the experiment. The latter parameter determines the cell radius $R$ (see the discussion in Ref. ${ }^{17}$ ). For the theoretical or simulational modeling those values were taken as input parameters and were not adjusted such as to fit the measured data.

Fig. 9 summarizes the results. It shows the osmotic coefficient $\phi$ of a PPP solution as a function of counterion concentration $c_{\mathrm{c}}$ as predicted by PoissonBoltzmann theory, the DHHC correlation-corrected treatment from Sec. 4.2, Molecular Dynamics simulations and experiment. 
To begin with, it is important to note the fine vertical scale. PoissonBoltzmann theory predicts $\phi$ to be smaller than 1 and vary roughly within the range $0.18 \ldots 0.22$. The measured values accumulate around 0.18 (iodine) and 0.16 (chlorine). Hence, the dominant change in $\phi$, a reduction by a factor of 5 , is correctly accounted for. However, on the enlarged scale of Fig. 9 it is visible that the measured values are systematically lower than the prediction, although still higher than the Manning limit $1 / 2 \xi$ of infinite dilution.

Both the correlation-corrected DHHC theory as well as the simulations, which in principle capture all kinds of ion correlations, show a decrease in the osmotic coefficient. Since these two totally different approaches agree so well, we believe that they indeed give a good description of the influence of correlations. However, they do not lower the osmotic coefficient sufficiently enough as to agree with the experimental results.

The deviation from the Poisson-Boltzmann curve increases for higher densities, which is true for the DHHC and simulational part as well as for the experiment. This appears plausible if one recalls that correlations become more important at higher densities. Unfortunately, the increasing systematic experimental errors in this regime are very difficult to quantify. Due to this fact, we give no significance to the apparent slight decrease of $\phi$ for the $\mathrm{I}^{-}$ions.

\section{Discussion}

The fact that Poisson-Boltzmann theory overestimates the osmotic coefficient as been observed previously. Careful studies of typical flexible polyelectrolytes in solution (see Ref. ${ }^{1,3}$ and further references given there) indicated that agreement of the Poisson-Boltzmann cell model and experimental data could only be approached if the charge parameter $\xi$ was renormalized to a higher value. The motivation given for this ad hoc modification was the assumption of a locally helical or wiggly main chain. Hence, the counterions "see" more charges per unit length, i.e., a macroion having a higher charge parameter. However, the results obtained for stiff-chain macroions ${ }^{17}$ show that the osmotic coefficient is lower than the Poisson-Boltzmann results even for systems where the local conformation of the macroion is absolutely rod-like, therefore this explanation can not be used. Also, the measured $\phi$ as a function of density has a functional form different from the Poisson-Boltzmann prediction, and no value of $\xi$ gives a curve that fits all data points.

Since Poisson-Boltzmann theory neglects all ion-ion correlations, it is tempting to assume that their incorporation into the theoretical treatment would resolve the discrepancy. However, our comparison shows that these correlational effects can be made responsible only for part of the deviations. Since the different approaches using a correlation-corrected density functional theory and Molecular Dynamics simulations agree very well with each other, we believe that the discrepancy between them and the experiment is not due to theoretical 
or simulational errors, but has to be looked for in the approximations underlying the cell model itself.

If there were any excess salt present in our system, the osmotic coefficient would be reduced. ${ }^{3,48,52}$ However, we performed numerical solutions of the Poisson-Boltzmann equation, taking into account various amounts of excess salt as well as the constraints due to the Donnan equilibrium, which showed two things (see Fig. 9): First, the functional form of $\phi\left(c_{\mathrm{c}}\right)$ is not compatible with a roughly constant value of the osmotic coefficient. Rather, it approaches the saltfree result at high $c_{\mathrm{c}}$ and drops strongly (below the Manning limit) at low $c_{\mathrm{c}}$. Second, our measurements at small counterion concentration are incompatible with the assumption of an excess salt concentration exceeding a few micromolar. In fact, the strong decrease of the osmotic coefficient at low concentrations, even below the Manning limiting value for the $\mathrm{Cl}^{-}$ions, can be explained by different very small excess salt concentrations for both experimental sequences (2 mikromol for $\mathrm{I}^{-}, 20$ mikromol for $\mathrm{Cl}^{-}$, compare Fig. 2). However, at our high concentrations, where the discrepancy is most apparent, any remaining excess salt is least relevant, and hence cannot explain neither the difference in the osmotic coefficient between $\mathrm{Cl}^{-}$and $\mathrm{I}^{-}$ions nor the decrease of $\phi$ compared to the PB prediction.

One obvious approximation of the cell model is the neglect of end effects of the rods by assuming them to be infinitely long. However, as we have discussed in Sec. 4.4, correcting this point would lead to an increased osmotic coefficient, since finite rods show less condensation (indeed, none in the limit of zero density). Moreover, the theoretical treatment of finite rods by Nyquist et $a l .{ }^{36}$ produces too large coefficients even at finite densities. Hence, finite size effects of the rods are unable to explain the additional reduction of the osmotic coefficient.

The theoretical approach mentioned in Sec. 4.3 yields a reduced osmotic coefficient. However, it is unable to predict values above the Manning limit, and shows a decrease of $\phi$ with increasing concentration, contrary to the experimental indications. The slight apparent decrease for the iodine is, if the errors are considered, not significant.

The remaining deficiency of the cell model is the neglect of any molecular detail, in particular: The solvent is described by a dielectric continuum. However, hydration effects are well known to be important in many circumstances ${ }^{53-55}$ and to depend on the particular objects to be hydrated. ${ }^{55}$ This of course applies to the counterions as well as to the charged groups of the macroion. Indeed, the difference between the osmotic coefficient measured for chlorine and iodine ions indicates that they are not just charged spheres. Similarly, the macroion is not just a charged rod.

Unfortunately, those last problems with the cell model are the hardest to deal with theoretically. Even predicting the direction into which changes are to be expected are very complicated. ${ }^{56}$ Obviously, much work still has to be done in order to arrive at an improved quantitative prediction of the osmotic 
coefficient of rodlike polyelectrolytes, since all the "obvious" modifications will not suffice, as we tried to show.

\section{Conclusion}

We tested the ability of the cell-model to explain the measured osmotic coefficient of solutions containing rodlike polyelectrolytes and monovalent counterions. We compared the Poisson-Boltzmann solution of the cell model, an improved local density approximation (DHHC), and simulational results of the model to recent experimental data.

Our findings are that the Poisson-Boltzmann solution of the cell model systematically overestimates the osmotic pressure. We showed that only a part of this discrepancy between theory and experiment is due to the neglect of correlations in the mean field approach, which will lower the osmotic pressure. We argued that other simple explanations like a locally curved main-chain conformation or the neglect of remaining excess salt do not apply here. We also outlined why dropping the assumption of infinite rods would in fact worsen the disagreement

We are led to the conclusion that the remaining discrepancy between experiment and a theoretical description of the system should be looked for on the level of molecular detail. This includes a better description of the solvent, hydration effects, or van der Waals forces. We are well aware of the fact that this requires formidable theoretical or numerical efforts, but we would like to stress that a full agreement between theory and experiment cannot and should not be expected on the level of a restricted primitive model for electrolytes, even if all correlations are properly taken into account. We believe that our comparison provides some evidence to this statement and thereby hope to motivate future theoretical and experimental work to resolve these issues.

\section{Acknowledgments}

The authors gratefully acknowledge financial support by the Deutsche Forschungsgemeinschaft, Schwerpunkt Polyelektrolyte, and a computer time grant hkf06 from NIC Jülich. We furthermore enjoyed discussions with M. Barbosa, P.L. Hansen, and R. Podgornik. 


\section{References}

${ }^{1}$ Mandel M., Polyelectrolytes. In: Encyclopedia of Polymer Science and Engineering, $2^{\text {nd }}$ edition, Eds.: Mark F. H., Bikales N. M., Overberger C. G., and Menges G., Wiley, New York, 1988, Vol. 11, 739.

${ }^{2}$ Oosawa F., Polyelectrolytes, Marcel Dekker, New York 1971.

${ }^{3}$ Katchalsky A., Pure Appl. Chem. 1971, 26, 327.

${ }^{4}$ Barat J. L. and Joanny, J.-F., In: Advances in Chemical Physics, Eds. Prigogine I. and Rice S. A., Vol. 94, 1, Wiley, 1996.

${ }^{5}$ Schmitz K. S., Macroions in solution and in colloidal suspension, VCH Publishers, New York 1993.

${ }^{6}$ Manning G. S., J. Chem. Phys. 1969, 51, 924, 934, 3249.

${ }^{7}$ Manning G. S., Ann. Rev. Phys. Chem. 1972, 23, 117.

${ }^{8}$ Manning G. S., In: Polyelectrolytes; Eds.: Sélegny, E., Mandel M., and Strauss U. P., Reidel, Dordrecht 1974, p. 9.

${ }^{9}$ Kern W., Z. Phys. Chem. 1938, A181, 268; Z. Phys. Chem. 1939, A184, 197.

${ }^{10}$ Auer H. E. and Alexandrowicz Z., Biopolymers 1969, 8, 1.

${ }^{11}$ Reddy M. and Marinsky J. A., J. Chem. Phys. 1970, 74, 3884.

${ }^{12}$ Takahashi A., Kato N., and Nagasawa M., J. Chem. Phys. 1970, 74, 944.

${ }^{13}$ Kozak D., Kristan J., and Dolar D., Z. Phys. Chem. Neue Folge 1971, 76, 85.

${ }^{14}$ Dolar D., In: Polyelectrolytes; Eds.: Sélegny E., Mandel M., and Strauss U. P., Reidel, Dordrecht 1974, p. 97.

${ }^{15}$ Kakehashi R., Yamazoe H., and Maeda, H., Colloid Polym. Sci. 1998, 276 , 28.

${ }^{16}$ Oppermann W. and Wagner M., Langmuir 1999, 15, 4089.

${ }^{17}$ Blaul J., Wittemann M., Ballauff M., and Rehahn M., J. Phys. Chem. B, 2000, 104, 7077 .

${ }^{18}$ Stevens, M.J. and Kremer, K., J. Chem. Phys. 1995, 103, 1669.

${ }^{19}$ Fuoss R. M., Katchalsky A., and Lifson S., Proc. Natl. Acad. Sci. USA 1951, 37,579 .

${ }^{20}$ Alfrey T., Berg P. W., and Morawetz H., J. Polym. Sci. 1951, 7, 543. 
${ }^{21}$ Le Bret M. and Zimm B. H., Biopolymers 1984, 23, 287.

${ }^{22}$ Mandel M., J. Phys. Chem. 1992, 96, 3934.

${ }^{23}$ Wennerström L., Jönsson B., and Linse P., J. Chem. Phys 1982, 76, 4665.

${ }^{24}$ Deserno M., Holm C., and May S., Macromolecules 2000, 33, 199.

${ }^{25}$ Marcus R. A., J. Chem. Phys. 1955, 23, 1057.

${ }^{26}$ Kjellander R. and Marcelja S., Chem. Phys. Lett. 1984, 112, 49; Chem. Phys. Lett. 1985, 114, 124(E); J. Chem. Phys. 1985, 82, 2122.

${ }^{27}$ Lozada-Cassou M., Saavedra-Barrera R., and Henderson D., J. Chem. Phys. 1982, 77, 5150. Gonzáles-Tovar E., Lozada-Cassou M., and Henderson D., J. Chem. Phys. 1985, 83, 361. Lozada-Cassou M. and Gonzáles-Tovar E., J. Phys. Chem. 1989, 93, 3761.

${ }^{28}$ Das T., Bratko D., Bhuiyan L. B., and Outhwaite C. W., J. Phys. Chem. 1995, 99, 410. Das T., Bratko D., Bhuiyan L. B., and Outhwaite C. W., J. Chem. Phys. 1997, 10\%, 9197, and further literature cited there.

${ }^{29}$ Nordholm S., Chem. Phys. Lett. 1984, 105, 302.

${ }^{30}$ Penfold R., Nordholm S., Jönsson B., and Woodward C. E., J. Chem. Phys. 1990, 92, 1915.

${ }^{31}$ Groot R. D., J. Chem. Phys. 1991, 95, 9191.

${ }^{32}$ Barbosa M. C., Deserno M., and Holm C., Euro. Phys. Lett. 2000, 52, 80.

${ }^{33}$ Deserno M., Physica A 2000, 278, 405.

${ }^{34}$ Kuhn P. S., Levin Y., and Barbosa M. C., Macromolecules 1998, 31, 8347.

${ }^{35}$ Levin, Y., Barbosa, M., J. Phys. II (Fr.) 1997, 7, 37.

${ }^{36}$ Nyquist R. M, Ha B.-Y., and Liu A., Macromolecules 1999, 32, 3481.

${ }^{37}$ Guldbrand L., Mol. Phys. 1989, 67, 217.

${ }^{38}$ Nilsson L. G., Guldbrand L., and Nordenskiöld L., Mol. Phys. 1991, 72, 117.

${ }^{39}$ Lyubartsev A. P. and Nordenskiöld L., J. Phys. Chem. 1995, 99, 10373; J. Phys. Chem. 1997, 101, 4335.

${ }^{40}$ Kassapidou K., Jesse W., Kuil M. E., Lapp A., Egelhaaf S., and van der Maarel J. R. C., Macromolecules 1997, 30, 2671.

${ }^{41}$ van der Maarel J. R. C. and Kassapidou K., Macromolecules 1998, 31, 5734. 
${ }^{42}$ Guilleaume B., Blaul J., Wittemann M., Rehahn M., and Ballauff M., J. Phys.: Cond. Mat. 2000, 12, A245.

${ }^{43}$ One may worry about impacts of the non-cylindrical geometry on the simulation. However, in the strongly charged regime most ions reside in a fairly close vicinity of the rod, where the potential is still cylindrical. It is those ions that will actually feel correlation effects and thereby trigger a readjustment of the whole ion profile as compared to Poisson-Boltzmann theory. Furthermore, the cell model partly relies on the assumption that the actual shape of the Wigner-Seitz-cell does not matter.

${ }^{44}$ Deserno M. and Holm C., J. Chem. Phys 1998, 109, 7678, 7694.

${ }^{45}$ Grest G. S. and Kremer K., Phys. Rev. A 1986, 33, 3628.

${ }^{46}$ Allen M. P. and Tildesley D. J., Computer Simulations of Liquids, Oxford Science Publications, Clarendon Press, 1997.

${ }^{47}$ Deserno M., Holm C., and Kremer K., In: Physical Chemistry of Polyelectrolytes, Ed. Radeva T., Marcel Decker, New York (in press); Deserno M. Ph.D. dissertation, Universität Mainz, 2000.

${ }^{48}$ Raspaud E., da Conceicao M., and Livolant F, Phys. Rev. Lett. 2000, 84, 2533.

${ }^{49}$ Donnan F. G., Chem. Rev. 1924, 1, 73.

${ }^{50}$ Usually the contribution to the total osmotic pressure coming from the counterions by far exceeds the macroion part. However, this may no longer hold if the ionic contribution almost cancels at low polyelectrolyte concentration due to the Donnan effect, and the macroion may reappear. Ref. ${ }^{48}$ speculates along similar lines.

${ }^{51}$ Galda P., Ph.D. Disseration, Universität Karlsruhe, 1994.

${ }^{52}$ Hansen P. L., Podgornik R., and Parsegian V. A., Preprint cond-mat/0008335, and personal communication.

${ }^{53}$ Conway B. E. and Ayranci E., Journal of Solution Chemistry 1999, 28, 163.

${ }^{54}$ Wilson J.E. Ansell S., Enderby J.E, and Neilson G.W., Chem Phys. Lett. 1997, 278, 21.

${ }^{55}$ Bieze T. W. N., Tromp R. H., van der Maarel J. R. C., van Strien M. H. J. M., Bellissent-Funel M. C., Neilson G. W., and Leyte J. C., J. Phys. Chem. 1994, 98, 4454 .

${ }^{56}$ Hünenberger, P.H., McCammon, J.A., J. Chem. Phys 1999, 110, 1856. 


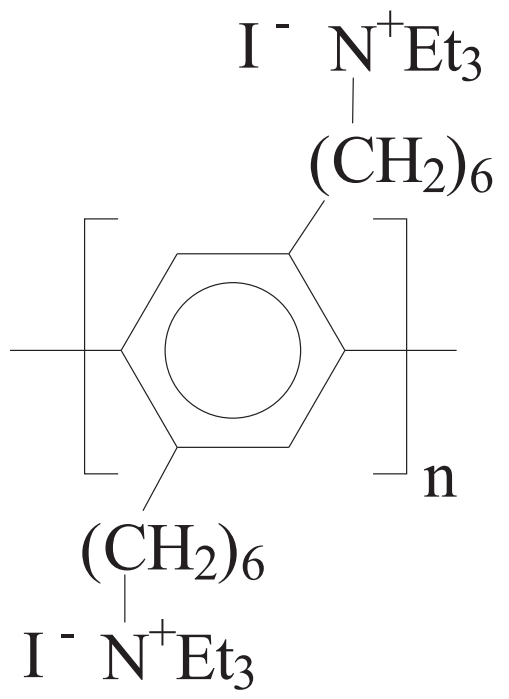

Figure 1: Constitution formula for poly(p-phenylene). The fully aromatic backbone exhibits an excellent chemical stability. Its uncharged precursor has a persistence length of approximately $22 \mathrm{~nm}$. The degree of polymerization used in the studies in Ref. ${ }^{17,42}$ was located between 20 and 40 . Therefore the contour length equals approximately one persistence length at most. 


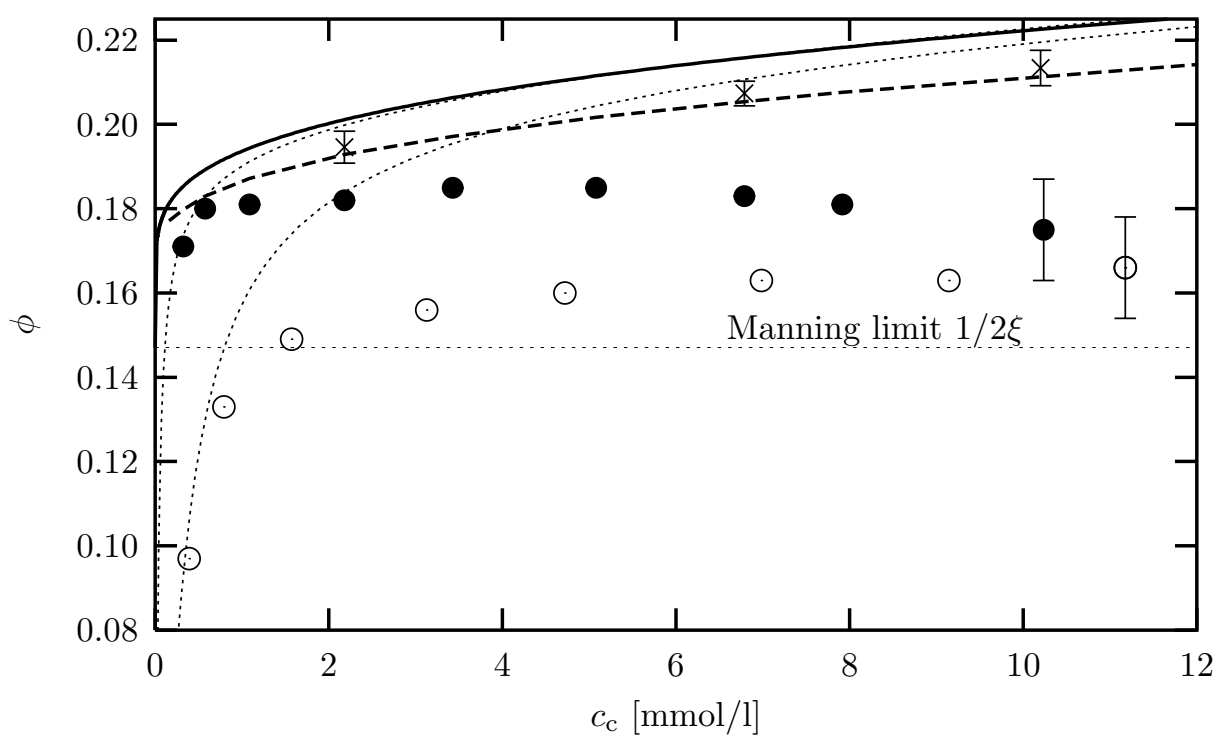

Figure 2: Osmotic coefficient as a function of counterion concentration $c_{\mathrm{c}}$ for the poly(p-phenylene) systems described in the text. The solid curve is the PB prediction of the cylindrical cell-model, the dashed curve is due to the correlation corrected PB theory from Ref. ${ }^{32}$ (see Sec. 4.2). The circles are experimental results using iodine (full) and chlorine (empty) counterions. Errors are of the order of $7 \%$ for all values, but only indicated for one measurement. The crosses originate from the MD simulations described in the text. Furthermore, the thin dashed lines are PB predictions assuming excess salt concentrations of 2 micromolar (upper) and 20 micromolar (lower), which are to be understood as crude estimates of the actual excess salt content. The Manning limiting value of $1 / 2 \xi$ is also indicated as a horizontal dashed line. 